\title{
Singularity-Free Dynamics Modeling and Control of Parallel Manipulators with Actuation Redundancy
}

\author{
Andreas Müller and Timo Hufnagel \\ University Duisburg-Essen, Chair of Mechanics and Robotics \\ Heilbronn University \\ Germany
}

\section{Introduction}

The modeling, identification, and control of parallel kinematics machines (PKM) have advanced in the last two decades culminating in successful industrial implementations. Still the acceptance of PKM is far beyond that of the well-established serial manipulators, however. This is mainly due to the limited workspace, the drastically varying static and dynamic properties, leading eventually to singularities, and the seemingly more complex control. Traditionally the number of inputs equals the mechanical degree-of-freedom (DOF) of the manipulator, i.e. the PKM is non-redundantly actuated.

Actuation redundancy is a means to overcome the aforementioned mechanical limitations. It potentially increases the acceleration capability, homogenizes the stiffness and manipulability, and eliminates input singularities, and thus increases the usable workspace as addressed in several publications as for instance Garg et al. (2009); Gogu (2007); Krut et al. (2004); Kurtz \& Hayward (1992); Lee et al. (1998); Nahon \& Angeles (1989); O’Brien \& Wen (1999); Wu et al. (2009).

These advantages are accompanied by several challenges for the dynamics modeling and for the PKM control. A peculiarity of redundantly actuated PKM is that control forces can be applied that have no effect on the PKM motion, leading to mechanical prestress that can be exploited for different second-level control tasks such as backlash avoidance and stiffness modulation Chakarov (2004); Cutkosky \& Wright (1986); Kock \& Schumacherm (1998); Lee et al. (2005); Müller (2006),Valasek et al. (2005). This also means that the inverse dynamics has no unique solution, which calls for appropriate strategies for redundancy resolution. The implementation of the corresponding model-based control schemes poses several challenges due to model uncertainties, the lack of globally valid parameterizations of the dynamics model, as well to the synchronization errors in decentralized control schemes calling for robust modeling and control concepts Müller (2011c); Müller \& Hufnagel (2011).

The basis for model-based control are the motion equations governing the PKM dynamics. Aiming on an efficient formulation applicable in real-time, the motion equations are commonly derived in terms of a minimum number of generalized coordinates that constitute a (local) parameterization of the configuration space Abdellatif et al. (2005); Cheng et al. (2003); Müller (2005); Nakamura \& Ghodoussi (1989); Yi et al. (1989). A well-known problem 
of this formulation is that these minimal coordinates are usually not valid on all of the configuration space, and configurations where the coordinates become invalid are called parameterization singularities. That is, it is not possible to uniquely determine any PKM configuration by one specific set of minimal coordinates. The most natural and practicable choice of minimal coordinates is to use the actuator coordinates. Then, the parameterization singularities are also input singularities. An ad hoc method to cope with this phenomenon is to switch between different minimal coordinates us discussed in Hufnagel \& Müller (2011); Müller (2011a). This is a computationally complex approach since it requires monitoring the numerical conditioning of the constraint equations, and the entire set of motion equation must be changed accordingly. Instead of using a minimal number of generalized coordinates together with the switching method, a formulation in terms of the entire set of dependent coordinates of the PKM was proposed in Müller (2011b;c). This gives rise to a large system of redundant equations. Despite the large system of motion equations this formulation is advantageous since it does not exhibit singularities, i.e. it is valid in all feasible configurations of the PKM. Now the peculiarity of RA-PKM is that input singularities can be avoided by means of the actuation redundancy. Consequently, the actuator coordinates represent a valid set of redundant parameters that may give rise to another system of redundant motion equations, but of smaller size, as discussed in the following.

In this chapter the applicability of the standard minimal coordinates formulation is discussed and an alternative formulation in terms of actuator coordinates is presented. The latter formulation is globally valid as long as the PKM does not encounter input singularities, which is the aim of applying redundant actuation. Upon this formulation an amended augmented PD (APD) and computed torque control (CTC) scheme is proposed. These model-based control schemes are shown to achieve exponentially stable trajectory tracking. Experimental results are shown for a prototype implementation of a 2-DOF redundantly actuated PKM.

\section{Kinematics of PKM with actuation redundancy}

Denoting the joint variables of the PKM with $q^{1}, \ldots, q^{n}$, the PKM configuration is represented by the joint coordinate vector $\mathbf{q} \in \mathbb{V}^{n}$. The kinematic loops of the PKM give rise to a system of $r$ geometric and kinematic constraints

$$
\begin{aligned}
& \mathbf{0}=\mathbf{h}(\mathbf{q}), \mathbf{h}(\mathbf{q}) \in \mathbb{R}^{r} \\
& \mathbf{0}=\mathbf{J}(\mathbf{q}) \dot{\mathbf{q}}, \mathbf{J}(\mathbf{q}) \in \mathbb{R}^{r, n} .
\end{aligned}
$$

The configuration space (c-space) of the PKM model is the set of admissible configurations, defined by the geometric constraints (1)

$$
V:=\left\{\mathbf{q} \in \mathbb{V}^{n} \mid \mathbf{h}(\mathbf{q})=\mathbf{0}\right\} .
$$

Presumed $\mathbf{J}$ has full rank, the PKM has the DOF $\delta:=n-r$. Consequently $\delta$ joint variables can be selected as independent coordinates representing a minimal set of generalized coordinates for the PKM and the (2) can be solved in terms of the corresponding independent velocities. Instead of using such independent minimal coordinates a solution can be expressed in terms of actuator coordinates.

To the actuated joints can be associated $m$ joint coordinates summarized in the vector $\mathbf{q}_{\mathrm{a}}$ so that the vector of joint coordinates can be rearranged as $\mathbf{q}=\left(\mathbf{q}_{\mathrm{p}}, \mathbf{q}_{\mathrm{a}}\right)$, where $\mathbf{q}_{\mathrm{p}}$ comprises the coordinates of passive coordinates. The constraints (2) can then be rearranged as

$$
\mathbf{J}_{\mathrm{p}} \dot{\mathbf{q}}_{\mathrm{p}}+\mathbf{J}_{\mathrm{a}} \dot{\mathbf{q}}_{\mathrm{a}}=\mathbf{0}
$$


with $r \times(n-m)$ matrix $\mathbf{J}_{\mathbf{p}}$.

The form (4) allows to identify two types of singularities. Assume that in regular configurations of the PKM (i.e. no c-space singularities) the constraint Jacobian $\mathbf{J}$ has full rank $r$. Further assume that the number $m$ of actuators is at least $\delta=n-r$. Then if rank $\mathbf{J}_{\mathrm{p}}=n-m$ and rank $\mathrm{J}_{\mathrm{a}}=\delta$, the PKM motion is always determined by the $m$ actuator coordinates and (4) can be resolved as $\dot{\mathbf{q}}_{\mathrm{p}}=-\mathbf{J}_{\mathrm{p}}^{+} \mathbf{J}_{\mathrm{a}} \dot{\mathbf{q}}_{\mathrm{a}}$ so that

$$
\dot{\mathbf{q}}=\widetilde{\mathbf{F}} \dot{\mathbf{q}}_{\mathrm{a}}
$$

with

$$
\widetilde{\mathbf{F}}=\left(\begin{array}{c}
-\mathbf{J}_{\mathrm{p}}^{+} \mathbf{J}_{\mathbf{a}} \\
\mathbf{I}_{m}
\end{array}\right)
$$

that satisfied $\mathbf{J} \widetilde{\mathbf{F}} \equiv \mathbf{0}$, where $\mathbf{J}_{\mathrm{p}}^{+}=\left(\mathbf{J}_{\mathrm{p}}^{T} \mathbf{J}_{\mathrm{p}}\right)^{-1} \mathbf{J}_{\mathrm{p}}^{T}$ is the left-pseudoinverse. In (5) it is assumed that $\dot{\mathbf{q}}_{\mathrm{a}}$ satisfies the constraints. Moreover, $\delta$ of them can serve as minimal coordinates.

Since actuation redundancy can eliminate input-singularities it is assumed in the following that the PKM does not encounter input-singularities in the relevant part of the workspace. A configuration $\mathbf{q}$ is called a c-space singularity if rank $\mathbf{J}$ changes in $\mathbf{q}$.

\section{Motion equations in actuator coordinates}

A PKM is a mechanism with kinematic loops, and the corresponding constraint forces are incorporated via the generalized constraint forces. The Lagrangian motion equations of the PKM can be represented in the standard form

$$
\mathbf{G}(\mathbf{q}) \ddot{\mathbf{q}}+\mathbf{C}(\mathbf{q}, \dot{\mathbf{q}}) \dot{\mathbf{q}}+\mathbf{Q}(\mathbf{q}, \dot{\mathbf{q}}, t)+\mathbf{J}^{T}(\mathbf{q}) \boldsymbol{\lambda}=\mathbf{u}
$$

where $\boldsymbol{\lambda}$ is the vector of Lagrange multipliers, $\mathrm{G}$ is the generalized mass matrix of the unconstrained system, $C \dot{q}$ represents generalized Coriolis and centrifugal forces, $\mathbf{Q}$ represents all remaining forces (possibly including EE loads), and $\mathbf{u}(t)$ are the generalized control forces. In the following a formulation of the equations of motion governing the PKM dynamics are derived in terms of actuator coordinates. Since the columns of $\widetilde{\mathbf{F}}$ constitute a basis for the null-space of $\mathbf{J}$, the generalized constraint reaction forces in (7) can be eliminated by premultiplication with $\widetilde{\mathbf{F}}^{T}$ at the same time reducing the number of equations. On a kinematic level the expression (5) and $\ddot{\mathbf{q}}=\dot{\widetilde{\mathbf{F}}} \dot{\mathbf{q}}_{\mathrm{a}}+\widetilde{\mathbf{F}} \ddot{\mathbf{q}}_{\mathrm{a}}$, where

$$
\dot{\widetilde{\mathbf{F}}}=\left(\begin{array}{c}
-\mathbf{J}_{\mathbf{p}}^{+} \dot{\mathbf{J}} \widetilde{\mathbf{F}} \\
\mathbf{0}_{m}
\end{array}\right),
$$

allow substitution of the generalized velocity and acceleration vector $\dot{\mathbf{q}}$ and $\ddot{\mathbf{q}}$, by actuator velocities and accelerations, respectively. This gives rise to the reduced system of motion equations

$$
\widetilde{\mathbf{G}}(\mathbf{q}) \ddot{\mathbf{q}}_{\mathrm{a}}+\widetilde{\mathbf{C}}(\mathbf{q}, \dot{\mathbf{q}}) \dot{\mathbf{q}}_{\mathrm{a}}+\widetilde{\mathbf{Q}}(\mathbf{q}, \dot{\mathbf{q}}, t)=\mathbf{c}
$$

with

$$
\widetilde{\mathbf{G}}:=\widetilde{\mathbf{F}}^{T} \mathbf{G} \widetilde{\mathbf{F}}, \widetilde{\mathbf{C}}:=\widetilde{\mathbf{F}}^{T}(\mathbf{C} \widetilde{\mathbf{F}}+\mathbf{G} \dot{\widetilde{\mathbf{F}}}), \widetilde{\mathbf{Q}}:=\widetilde{\mathbf{F}}^{T} \mathbf{Q}
$$

and $\mathbf{c}$ represents the actuator forces being part of the overall vector $\mathbf{u}$.

This is a reduced system of $m$ equations. It is crucial to notice that only $\delta$ of these $m$ equations are independent. That is, if the PKM is redundantly actuated $(m>\delta)$, the projected mass 
matrix is $\widetilde{\mathbf{G}}$ is singular. However, the advantage of the formulation (9) is that it already represents a solution for the inverse dynamics problem, in contrast to the minimal coordinate formulations Müller (2005). This will be beneficial for the model-based control.

\section{Parameterization singularities of non-redundantly actuated PKM}

The selection of actuator coordinates induces a parameterization of the PKM configuration. Commonly a minimal set of $\delta$ independent coordinates, denoted $\mathbf{q}_{2}$, are selected giving rise to $\delta$ motion equations. It is well-known, however, that such minimal coordinates are not globally valid in the sense that there are configuration where a particular set of of coordinates does not uniquely determine the PKM motion. Such configurations are called parameterization singularities. For non-redundantly actuated PKM $\delta=m$ actuator coordinates are usually taken as independent coordinates, so that $\mathbf{q}_{2}=\mathbf{q}_{\mathrm{a}}$ and the parameterization singularities are exactly the input singularities. In these cases $\mathbf{J}_{\mathbf{a}}$ in (4) is rectangular and invertible as long as the non-redundantly actuated PKM does not encounter input singularities.

To explain this phenomenon consider the planar $2 \underline{R} R R / \underline{R} R$ PKM shown in figure 1 . This system has a DOF $\delta=2$, but is actuated by the $m=3$ actuators at the base joints. Hence it is redundantly actuated with a degree of redundancy of $\rho=m-\delta=1$. This PKM is naturally parameterized in terms of two of these three actuator coordinates. Now, as outlined above, there are configurations where the PKM motion is cannot be prescribed by $\delta=2$ coordinates. These parameterization-singularities can be observed if the joint angles of two of these actuators are used as independent coordinates. Figure 2 shows the EE locus corresponding to the input-singularities for three different choices of independent coordinates. The EE traces of the singularities in workspace are the coupler curves of the 4-bar mechanism formed by fixing the middle joint so that the middle links keep aligned.

To cope with the lack of a globally valid parameterization of the PKM configuration a switching method for RA-PKM was proposed in Hufnagel \& Müller (2011). The basic idea of this method is to switch to a different set of $\delta$ (actuator) coordinates whenever the current set fails. The drawback of this method is that for each set of independent coordinates different set motion equations must be invoked, which increases the implementation effort. Ideally

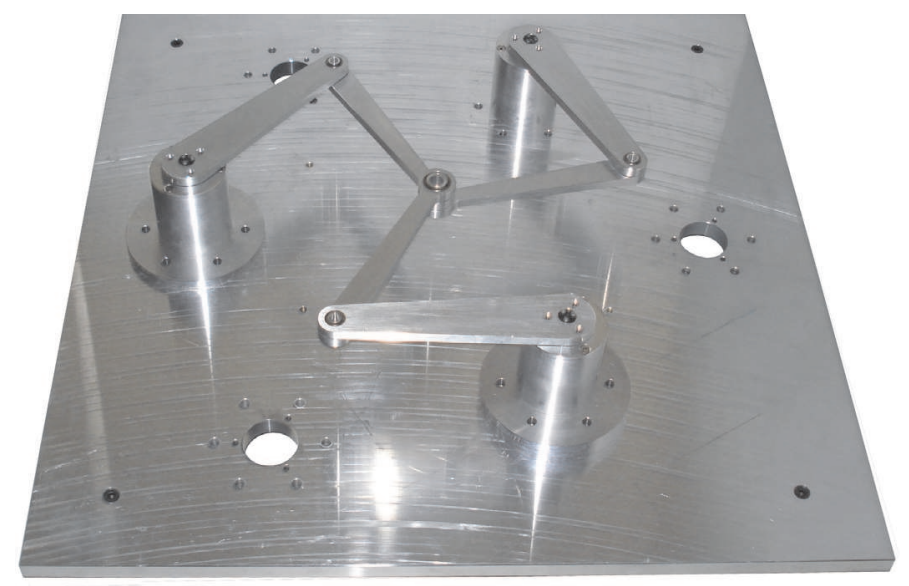

Fig. 1. Prototype of a planar $2 \underline{R} R R / \underline{R} R$ RA-PKM. 


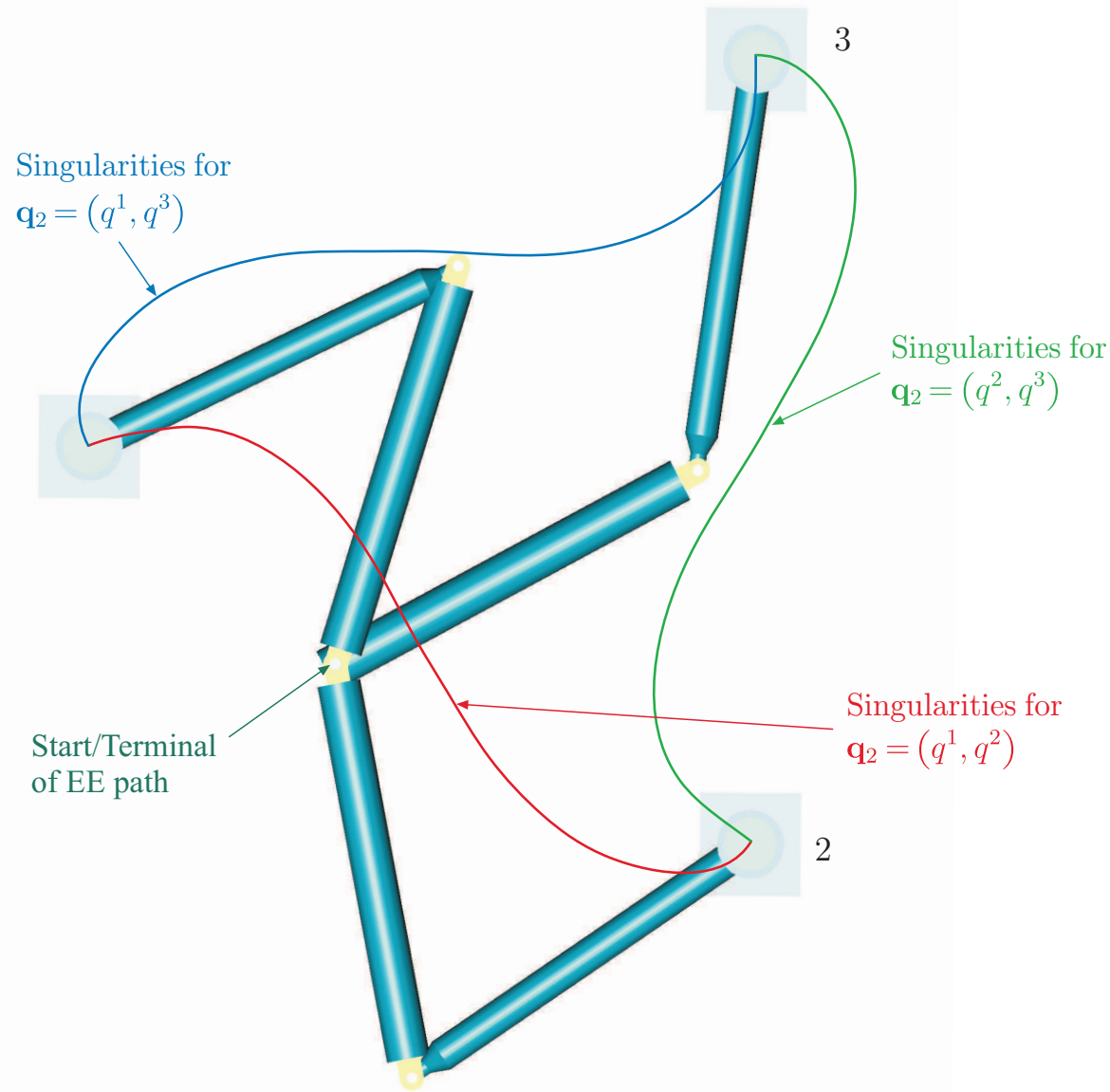

Fig. 2. Different input singularities if the 2 DOF $2 \underline{R} R / \underline{R} R R$ PKM is non-redundantly actuated. The mechanism shown in color is the equivalent non-redundantly actuated mechanisms being instantaneously in an input-singularity.

a formulation should be used that is free from any parameterization singularities within the range of motion of the PKM. It often suggested to use EE coordinates as independent generalized coordinates, so that (9) would govern the PKM dynamics in workspace. It turns out, however, that even this choice suffers from parameter singularities.

Now the main motivation for introduction of redundant actuation is that most, or possibly all, of the input singularities of the non-redundant PKM can be eliminated. Assumption that the RA-PKM does not possess input-singularities, i.e. it can always be controlled by some $\delta$ out of the $m$ actuator coordinates, the $m$ input coordinates $\mathbf{q}_{\mathbf{a}}$ constitute feasible coordinates to parameterize the PKM motion. Hence the system (9) is globally valid for the entire motion 
range. In summary, the redundant formulation (9) is globally valid in the entire motion range of a RA-PKM where it does not exhibit input-singularities. If $m=\delta$, i.e. non-redundant actuation, the formulation (9) reduces to the classical formulation in minimal coordinates Müller (2005).

\section{Model-based control schemes in redundant actuator coordinates}

While the solution of the inverse dynamics problem of RA-PKM in minimal coordinates involves the pseudoinverse of the $m \times \delta$ control matrix, the formulation (9) in redundant actuator coordinates is already an inverse dynamics solution that can be immediately employed for the feedforward. Therewith an APD control scheme can be introduced as

$$
\begin{aligned}
\mathbf{c} & =\widetilde{\mathbf{G}}(\mathbf{q}) \ddot{\mathbf{q}}_{\mathrm{a}}^{\mathrm{d}}+\widetilde{\mathbf{C}}(\dot{\mathbf{q}}, \mathbf{q}) \dot{\mathbf{q}}_{\mathrm{a}}^{\mathrm{d}}+\widetilde{\mathbf{Q}}(\mathbf{q}, \dot{\mathbf{q}}, t)-\mathbf{K}_{\mathrm{P}} \mathbf{e}_{\mathrm{a}}-\mathbf{K}_{\mathrm{D}} \dot{\mathbf{e}}_{\mathrm{a}} \\
& =\widetilde{\mathbf{F}}^{T}\left(\mathbf{G}(\mathbf{q}) \ddot{\mathbf{q}}_{\mathrm{a}}^{\mathrm{d}}+\mathbf{C}(\mathbf{q}, \dot{\mathbf{q}}) \dot{\mathbf{q}}_{\mathrm{a}}^{\mathrm{d}}+\mathbf{Q}(\mathbf{q}, \dot{\mathbf{q}}, t)\right)-\mathbf{K}_{\mathrm{P}} \mathbf{e}_{\mathrm{a}}-\mathbf{K}_{\mathrm{D}} \dot{\mathbf{e}}_{\mathrm{a}}
\end{aligned}
$$

where $\mathbf{q}_{a}^{\mathrm{d}}(t)$ is the target trajectory and $\mathbf{e}_{\mathrm{a}}:=\mathbf{q}_{\mathrm{a}}-\mathbf{q}_{\mathrm{a}}^{\mathrm{d}}$ is the tracking error. The gain matrices $\mathbf{K}=\operatorname{diag}\left(K_{1}, \ldots, K_{m}\right)$ in the linear feed-back measure the errors in the $m$ actuator coordinates.

Further a CTC scheme in terms of actuator coordinates can be introduced as

$$
\begin{aligned}
\mathbf{c} & =\widetilde{\mathbf{G}}(\mathbf{q}) \mathbf{v}_{\mathrm{a}}+\widetilde{\mathbf{C}}(\dot{\mathbf{q}}, \mathbf{q}) \dot{\mathbf{q}}_{\mathrm{a}}+\widetilde{\mathbf{Q}}(\mathbf{q}, \dot{\mathbf{q}}, t) \\
& =\widetilde{\mathbf{F}}^{T}\left(\mathbf{G}(\mathbf{q}) \mathbf{v}_{\mathrm{a}}+\mathbf{C}(\mathbf{q}, \dot{\mathbf{q}}) \dot{\mathbf{q}}_{\mathrm{a}}+\mathbf{Q}(\mathbf{q}, \dot{\mathbf{q}}, t)\right)
\end{aligned}
$$

with $\mathbf{v}_{\mathrm{a}}=\ddot{\mathbf{q}}_{\mathrm{a}}-\mathbf{K}_{\mathrm{P}} \mathbf{e}_{\mathrm{a}}-\mathbf{K}_{\mathrm{D}} \dot{\mathbf{e}}_{\mathrm{a}}$.

It is crucial that these control schemes lead to exponentially stable trajectory tracking. This property can be shown as for the minimal coordinates formulation by projection of the error dynamics to a $\delta$ subspace of the c-space $V$. Notice that in $(11)_{2}$ and $(12)_{2}, \mathbf{q}_{\mathrm{a}}^{\mathrm{d}}(t)$ and $\mathbf{q}_{\mathrm{a}}(t)$ are presumed to satisfy the constraints. If this is not ensured, when using measured values for instance, the formulation $(11)_{1}$ and $(12)_{1}$, respectively, is to be used.

At this point it should be remarked that the errors of all $m>\delta$ actuator coordinates must be used in the linear feedback. If only $\delta$ independent actuator coordinates are used, the feedback term does not account for the overall error in configurations where the motion is not uniquely determined by these $\delta$ actuator motions, i.e. in parameterization-/input-singularities of the non-redundantly actuated PKM. On the other hand the redundant feedback causes counteraction of the $m$ actuators since only $\delta$ actuator coordinates are independent but the $m$ feedback commands are not.

While the actuator coordinate formulation offers globally valid motion equations it does not involve the components of the control forces that correspond to the null-space of the control matrix as the inverse dynamics solution in minimal coordinates does.

\section{Experimental results}

The prototype of the planar 2 DOF $2 \underline{R} R R / \underline{R} R$ PKM in figure 1 has been used as testbed for the proposed control schemes in redundant actuator coordinates and the classical formulation in minimal coordinate. The testbed is equipped with a dSPACE DS1103 real-time system operating with a sampling rate of $2.5 \mathrm{kHz}$. The controller gains were set to $K_{\mathrm{P}}=500$ and $K_{\mathrm{D}}=8$, in accordance to the actuator dynamics. The three base joints are actuated by Maxon Re30 DC motors. They are mounted at the vertices of an equilateral triangle with $400 \mathrm{~mm}$ 


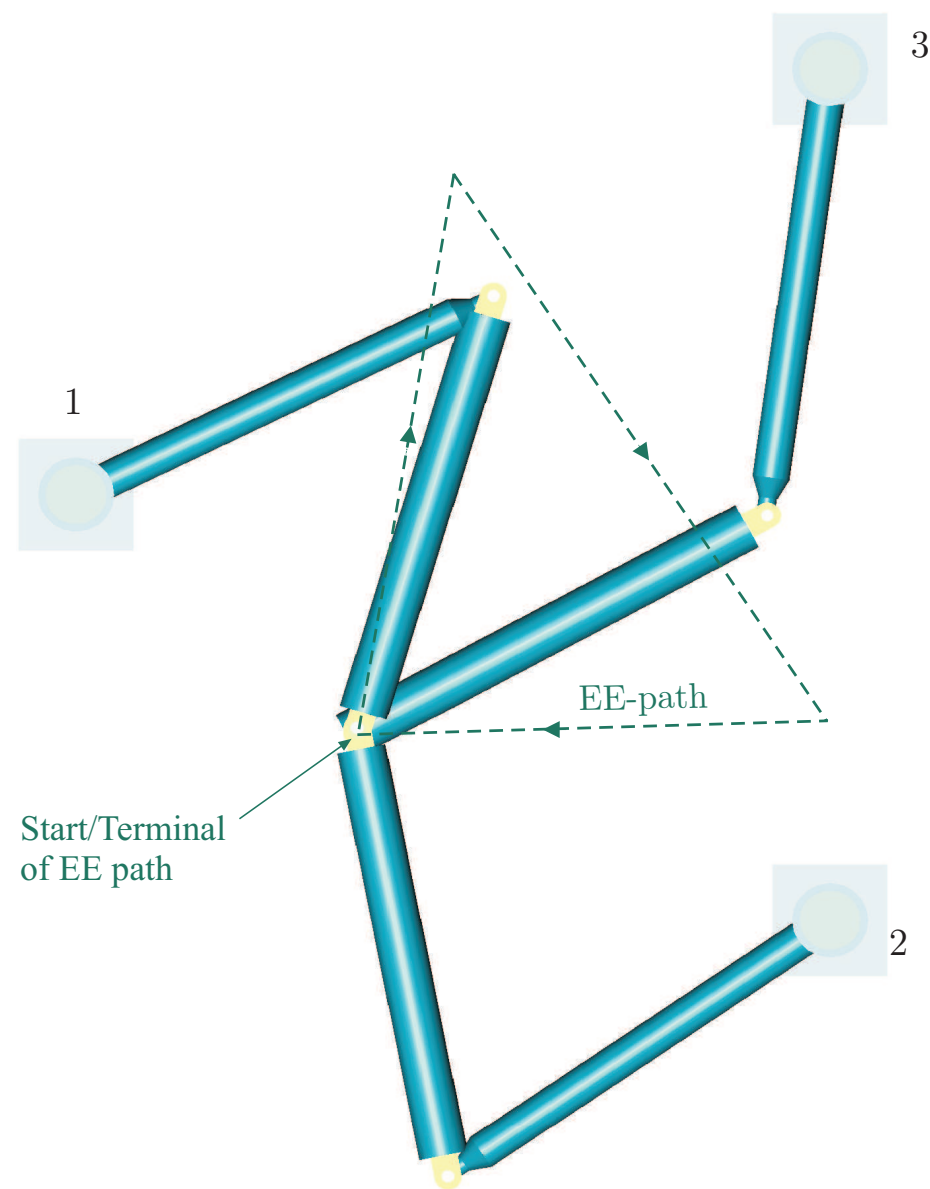

Fig. 3. EE-path and singular curves of the 2DOF RA-PKM.

lateral lengths. Each arm segment has a length of $200 \mathrm{~mm}$, and the total weight of one arm is $134 \mathrm{~g}$. The motion equations (7) are derived in terms of relative coordinates by opening the two kinematic loops. This gives rise to a PKM model (7) comprising $n=6$ equations in terms of the $n=6$ joint angles subject to $r=4$ cut-joint constraints. Hence the DOF of the PKM is $\delta=2$. the formulation (9) yields $m=3$ equations in terms of the redundant actuator coordinates (of which $\delta=2$ are independent). In the experiment the manipulator is controlled along the EE-path in figure 3. Denote with $q^{1}, q^{2}, q^{3}$ the joint angles of the actuated base joints so that $\mathbf{q}_{a}=\left(q^{1}, q^{2}, q^{3}\right)$. The joint trajectory is determined from the EE-path by solving the inverse kinematics where velocity and acceleration limits are taken into account. The EE path passes all singularity loci shown in 2. That is, a non-redundantly actuated PKM, of which only $\delta=2$ joints are actuated, exhibits input-singularities for any combination of two actuator coordinates. 


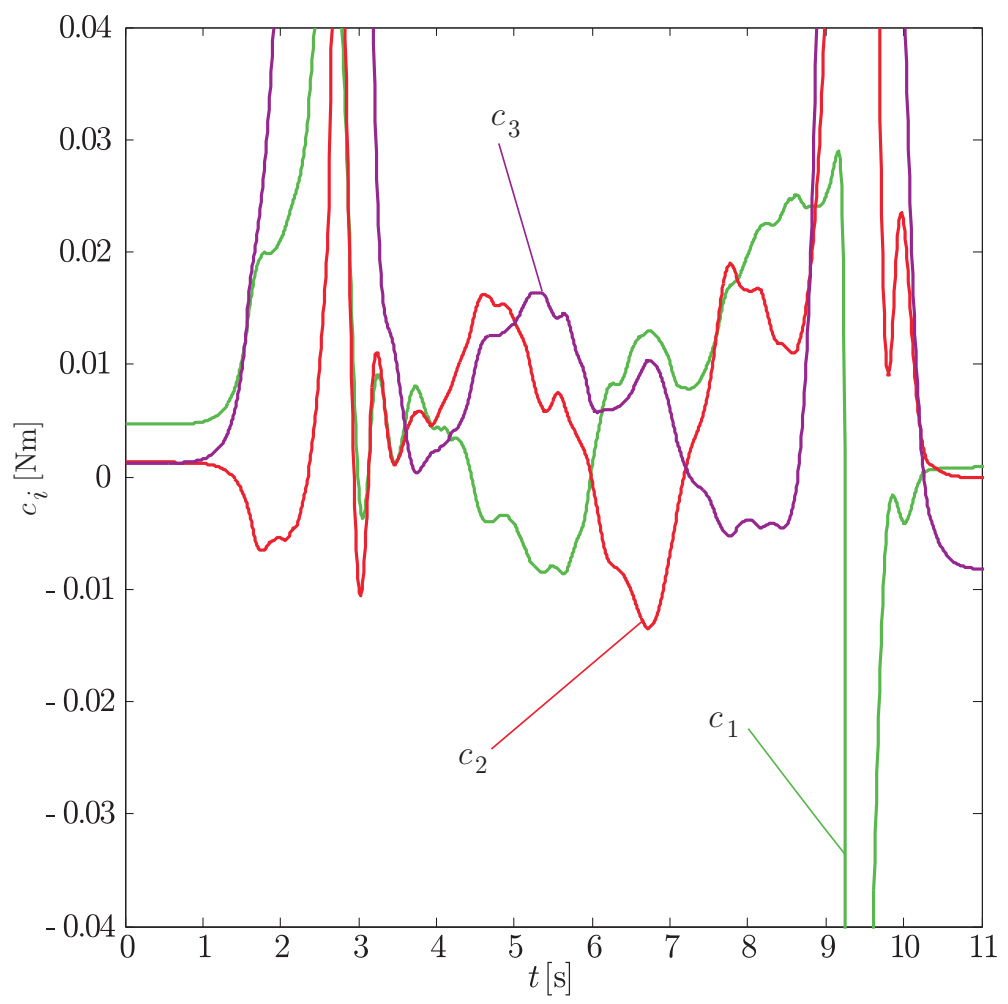

Fig. 4. Actuator torques computed from the CTC in minimal cooridnates if $\mathbf{q}_{\mathrm{a}}=\left(q^{1}, q^{2}\right)$.

In order to show the effect of parameter singularities of the minimal coordinate formulation figure 4 shows the computed control commands when only the two coordinates $\mathbf{q}_{\mathrm{a}}=\left(q^{1}, q^{2}\right)$ are used in (9), which corresponds to a non-redundantly actuated PKM. Since for this EE path the PKM has to cross the singularity curve for this combination (red curve in figure 2) once at the start and once just before the end of the EE-trajectory the control torques tend to infinity at these points. Consequently at these points the model does not admit to compute any sensible control torques and leads to instabilities.

In contrast, when controlling the RA-PKM using the CTC (12) in redundant actuator coordinates leads to the drive torques in figure 5. Figure 6 sows the corresponding joint tracking errors. Apparently the redundant coordinate formulation achieves a smooth motion and torque evolution unaffected by any singularities.

In this experiment it is clearly visible that there are non-zero drive torques even if the RA-PKM is not moving. This is a peculiar phenomenon that can only be observed in RA-PKM. It can partially be attributed to the interplay of measurement errors and finite encoder resolutions with actuation redundancy. Due to the actuation redundancy the control forces are not independent so that the RA-PKM attains a configuration that is not determined by the measurement error (as for non-redundant actuation) but by the static equilibrium of the control torques that do not affect the RA-PKM motion, but rather lead to internal prestress. This is a general problem for RA-PKM that was addressed in Müller \& Hufnagel (2011). 


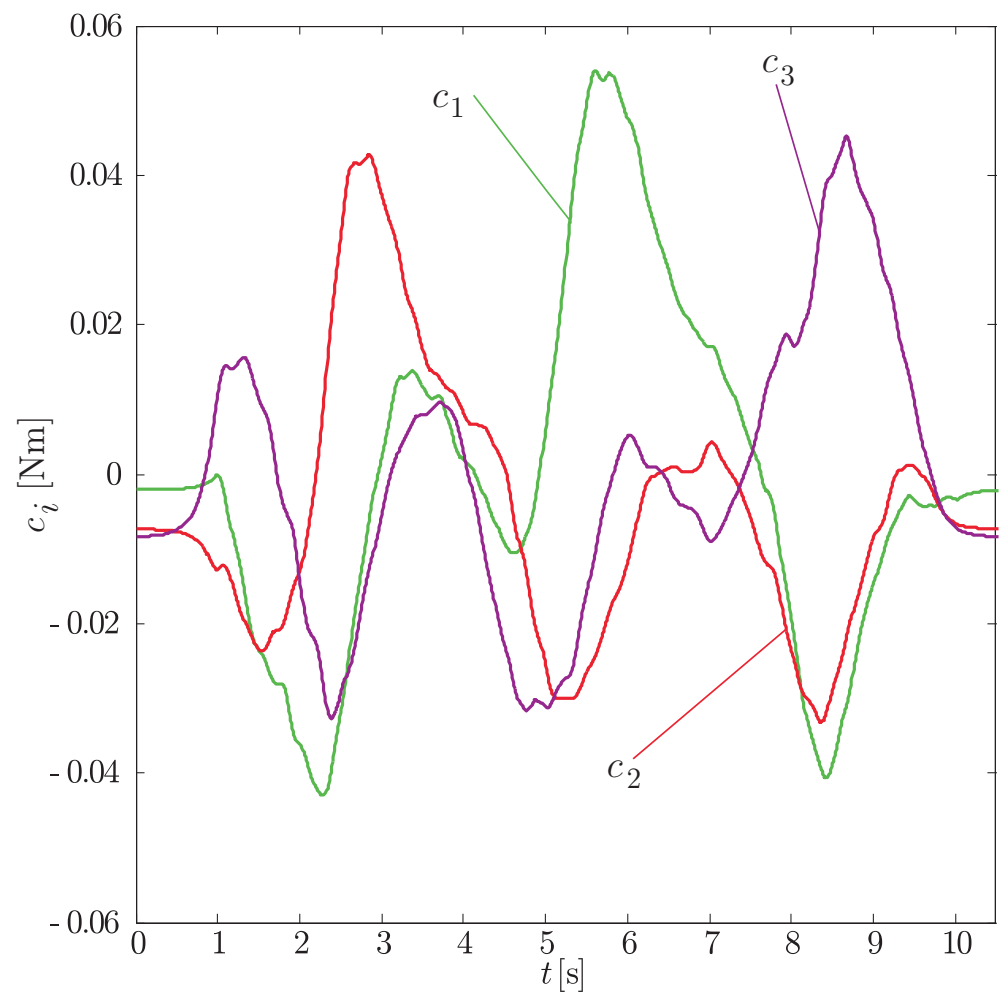

Fig. 5. Actuator torques when the RA-PKM is controlled along the EE-path of figure 3 by the CTC in redundant coordinates. 


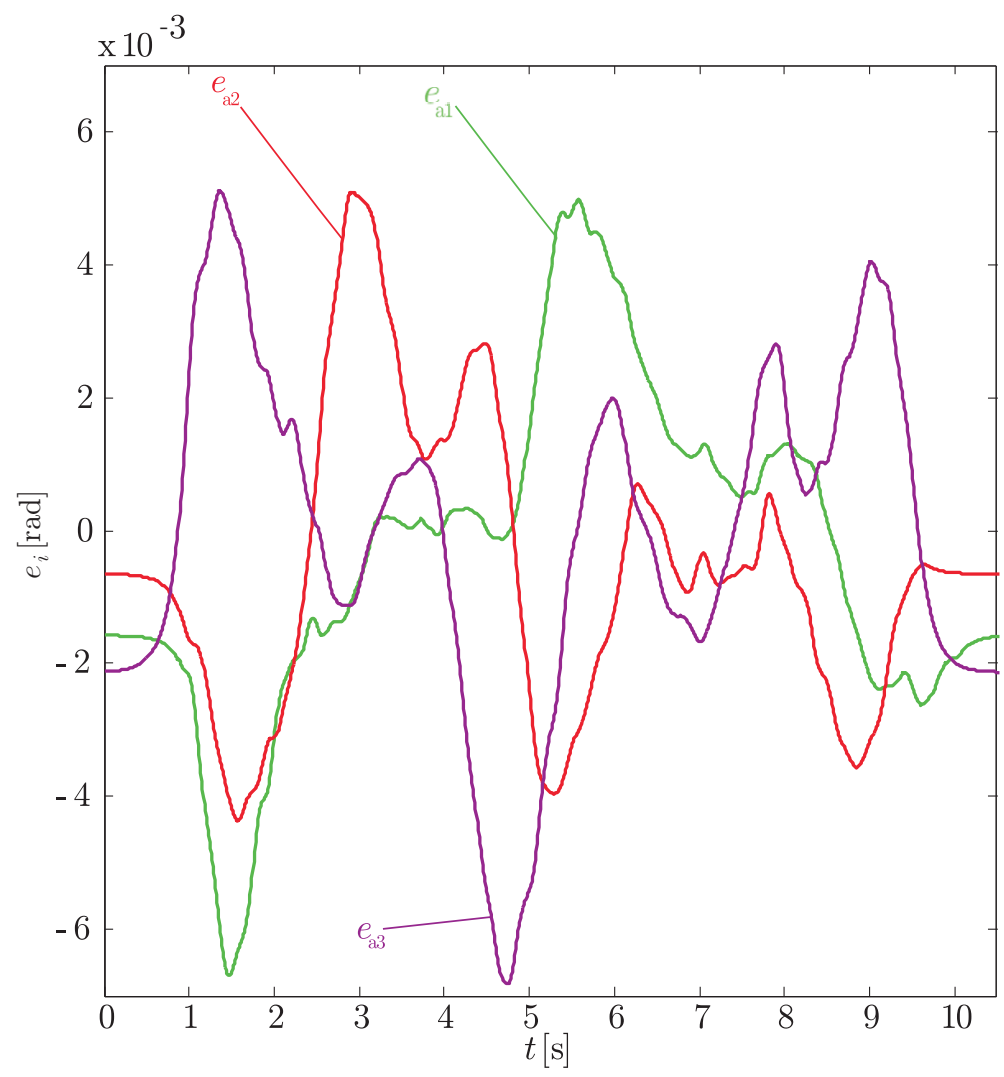

Fig. 6. Joint tracking errors when the RA-PKM is controlled by the CTC in redundant coordinates. 


\section{Summary}

PKM are commonly characterized by inhomogeneous distribution of kinematic and dynamic properties within the work space, and eventually the existence of input-singularities. Actuation redundancy allows to eliminate these singularities, and thus to extend the usable workspace. The PKM motion equations are commonly formulated using a set of minimal coordinates. Moreover, actuator coordinates are usually used as minimal coordinates. Thus input-singularities are also parameterization-singularities of the PKM model, which is critical for any model-based control. In this chapter an alternative formulation of motion equations in terms of redundant actuator coordinates has been proposed, and the corresponding amended form of an augmented PD and computed torque control scheme were presented. The applicability of the proposed methods is confirmed by the experimental results for a planar redundantly actuated PKM.

\section{References}

H. Abdellatif et al. (2005) High Efficient Dynamics Calculation Approach for Computed-Force Control of Robots with Parallel Structures, IEEE Conference on Decision and Control and European Control Conference (CDC-ECC), 12-15 Dec. 2005, pp. 2024-2029

H. Asada \& J.J. E. Slotine (1986) Robot Analysis and Control. New York, Wiley

D. Chakarov (2004) Study of the antagonistic stiffness of parallel manipulators with actuation redundancy, Mech. Mach. Theory, Vol. 39, 2004, pp. 583-601

H. Cheng et al. (2003) Dynamics and Control of Redundantly Actuated Parallel Manipulators, IEEE/ASME Trans. on Mechatronics, Vol. 8, no. 4, 2003, pp. 483-491

M.R. Cutkosky \& P.K. Wright (1986) Active Control of a Compliant Wrist in Manufacturing Tasks, ASME J. Eng. for Industry, Vol. 108, No. 1, 1986, pp. 36-43

Garg, V.; Noklely, S.B. \& Carretero, J.A. (2009). Wrench capability analysis of redundantly actuated spatial parallel manipulators, Mech. Mach. Theory, Vol. 44, No. 5, 2009, pp: 1070-1081

Gogu, G. (2007). Fully-isotropic redundantly-actuated parallel wrists with three degrees of freedom, Proc. ASME Int. Design Eng. Tech. Conf. (IDETC), Las Vegas, NV, 2007, DETC2007-34237

T. Hufnagel \& A. Müller (2011) A Realtime Coordinate Switching Method for Model-Based Control of Parallel Manipulators, ECCOMAS Thematic Conference on Multibody Dynamics, Juli 4-7, 2011, Brussels, Belgium

S. Kock \& W. Schumacher (1998) A parallel x-y manipulator with actuation redundancy for high-speed and active-stiffness applications, Proc. IEEE Int. Conf. Robot. Autom. (ICRA), Leuven, pp. 2295-2300

Krut, S.; Company, O. \& Pierrot, F. (2004) Velocity performance indicies for parallel mechanisms with actuation redundancy, Robotica, Vol. 22, 2004, pp. 129-139

Kurtz, R.; Hayward, V. (1992) Multiple-goal kinematic optimization of a parallel spherical mechanism with actuator redundancy, IEEE Trans. on Robotics Automation, Vol. 8. no. 5, 1992, pp. 644-651

Lee, J.H.; Li, B.J.; Suh, H. (1998) Optimal design of a five-bar finger with redundant actuation, Proc. IEEE Int. Conf. Rob. Aut. (ICRA), Leuven, 1998, 2068-2074

S.H. Lee et al. (2005) Optimization and experimental verification for the antagonistic stiffness in redundantly actuated mechanisms: a five-bar example, Mechatronics Vol. 15, 2005, pp. $213-238$ 
Müller, A. (2005). Internal Prestress Control of redundantly actuated Parallel Manipulators Its Application to Backlash avoiding Control, IEEE Trans. on Rob., pp. 668 - 677, Vol. 21, No. 4, 2005

A. Müller (2006). Stiffness Control of redundantly actuated Parallel Manipulators, Proc. IEEE Int. Conf. Rob. Automat. (ICRA), Orlando, May 15-19, 2006, pp. 1153 - 1158

A. Müller (2011). A Robust Inverse Dynamics Formulation for Redundantly Actuated PKM, Proc. 13th World Congress in Mechanism and Machine Science, Guanajuato, Mexico, 19-25 June 2011

A. Müller (2011). Motion Equations in Redundant Coordinates with Application to Inverse Dynamics of Constrained Mechanical Systems, Nonlinear Dynamics, DOI $10.1007 / \mathrm{s} 11071-011-0165-5$

A. Müller (2011) Robust Modeling and Control Issues of Parallel Manipulators with Actuation Redundancy, in A. Müller (editor): Recent Advances in Robust Control - Volume 1: Theory and Applications in Robotics and Electromechanics, InTech, Vienna, Austria, 2011

A. Müller, T. Hufnagel (2011) Adaptive and Singularity-Free Inverse Dynamics Models for Control of Parallel Manipulators with Actuation Redundancy, 8th International Conference on Multibody Systems, Nonlinear Dynamics, and Control, ASME 2011 International Design Engineering Technical Conferences, August 28-31, 2011, Washington, DC

A. Müller, T. Hufnagel (2011) A Projection Method for the Elimination of Contradicting Control Forces in Redundantly Actuated PKM, IEEE Int. Conf. Rob. Automat. (ICRA), May 9-13, 2011, Shanghai, China

Nahon, M.A.; Angeles, J. (1989) Force optimization in redundantly-actuated closed kinematic chains, Proc. IEEE Int. Conf. Rob. Automat. (ICRA), Scottsdale, USA, May 14-19, 1989, pp. 951956

Y. Nakamura \& M. Ghodoussi (1989) Dynamics Computation of Closed-Link Robot Mechanisms with Nonredundant and Redundant Actuators, IEEE Tran. Rob. and Aut., Vol. 5, No. 3, 1989, pp. 294-302

O'Brien, J.F.; Wen, J.T. (1999) Redundant actuation for improving kinematic manipulability, Proc. IEEE Int. Conf. Robotics Automation, 1999, pp. 1520-1525

Valasek, M. et al. (2005) Design-by-Optimization and Control of Redundantly Actuated Parallel Kinematics Sliding Star, Multibody System Dynamics, Vol. 14, no. 3-4, 2005, 251-267

$\mathrm{Wu}$, J. et al. (2009) Dynamics and control of a planar 3-DOF parallel manipulator with actuation redundancy, Mech. Mach. Theory, Vol. 44, 2009, pp. 835-849.

B.Y. Yi et al. (1989) Open-loop stiffness control of overconstrained mechanisms/robot linkage systems, Proc. IEEE Int. Conf. Robotics Automation, Scottsdale, 1989, pp. 1340-1345 


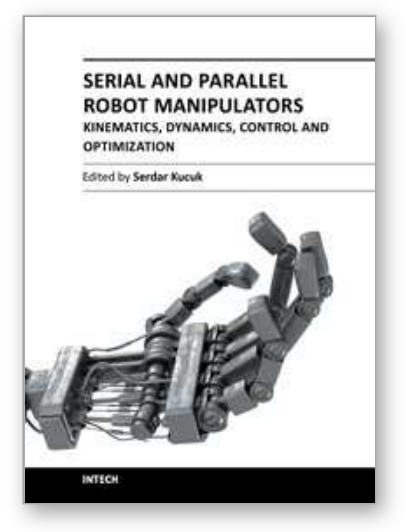

\section{Serial and Parallel Robot Manipulators - Kinematics, Dynamics, Control and Optimization}

Edited by Dr. Serdar Kucuk

ISBN 978-953-51-0437-7

Hard cover, 458 pages

Publisher InTech

Published online 30, March, 2012

Published in print edition March, 2012

The robotics is an important part of modern engineering and is related to a group of branches such as electric \& electronics, computer, mathematics and mechanism design. The interest in robotics has been steadily increasing during the last decades. This concern has directly impacted the development of the novel theoretical research areas and products. This new book provides information about fundamental topics of serial and parallel manipulators such as kinematics \& dynamics modeling, optimization, control algorithms and design strategies. I would like to thank all authors who have contributed the book chapters with their valuable novel ideas and current developments.

\section{How to reference}

In order to correctly reference this scholarly work, feel free to copy and paste the following:

Andreas Müller and Timo Hufnagel (2012). Singularity-Free Dynamics Modeling and Control of Parallel Manipulators with Actuation Redundancy, Serial and Parallel Robot Manipulators - Kinematics, Dynamics, Control and Optimization, Dr. Serdar Kucuk (Ed.), ISBN: 978-953-51-0437-7, InTech, Available from: http://www.intechopen.com/books/serial-and-parallel-robot-manipulators-kinematics-dynamics-control-andoptimization/singularity-free-dynamics-modeling-and-control-of-parallel-manipulators-with-actuationredundancy

\section{INTECH}

open science | open minds

\author{
InTech Europe \\ University Campus STeP Ri \\ Slavka Krautzeka 83/A \\ 51000 Rijeka, Croatia \\ Phone: +385 (51) 770447 \\ Fax: +385 (51) 686166 \\ www.intechopen.com
}

\author{
InTech China \\ Unit 405, Office Block, Hotel Equatorial Shanghai \\ No.65, Yan An Road (West), Shanghai, 200040, China \\ 中国上海市延安西路65号上海国际贵都大饭店办公楼 405 单元 \\ Phone: +86-21-62489820 \\ Fax: $+86-21-62489821$
}


(C) 2012 The Author(s). Licensee IntechOpen. This is an open access article distributed under the terms of the Creative Commons Attribution 3.0 License, which permits unrestricted use, distribution, and reproduction in any medium, provided the original work is properly cited. 